\title{
Nanofluid natural convection around a cylinder by BEM
}

\author{
J. Ravnik ${ }^{1}$, L. Škerget ${ }^{1}$ \& B. W. Yeigh ${ }^{2}$ \\ ${ }^{1}$ Faculty of Mechanical Engineering, University of Maribor, Slovenia \\ ${ }^{2}$ University of Washington, USA
}

\begin{abstract}
In this paper we focus on the determination of vorticity boundary conditions for the solution of Navier-Stokes equations in velocity-vorticity form. Boundary element method is used to calculate the vorticity boundary conditions on an arbitrary curved surface. The method is used in an in-house fluid and heat transfer solver.

Results of simulations of flow and heat transfer of nanofluids are presented. We consider a heated elliptical cylinder in a cooled cubic enclosure where natural convection develops. Steady laminar regime is considered with Rayleigh number values up to a million. $\mathrm{Al}_{2} \mathrm{O}_{3}$ nanofluid is considered, as well as pure water for validation purposes. Properties of the nanofluid are considered to be constant throughout the domain and are estimated using models for different nanoparticle volume fractions ( 0.1 and 0.2 ).

The results show highest heat transfer enhancement in the conduction dominated flow regime, where the enhanced thermal properties of nanofluids play an important role. When convection is the dominant heat transfer mechanism, using nanofluids yields a smaller increase in heat transfer efficiency.

Keywords: nanofluids, boundary element method, vorticity boundary conditions, heat transfer, velocity-vorticity.
\end{abstract}

\section{Introduction}

Cooling is one of the major challenges in development of efficient devices. Natural convection is used to design many devices, for example, heat exchangers and electronics coolers. Choice of a working fluid is very important, as its thermal properties determine heat transfer characteristics. As thermal conductivity of water, oil and other working fluids are low, Choi [1] introduced nanofluids. 
Nanofluid is a suspension consisting of uniformly dispersed and suspended nanometre-sized $(10-50 \mathrm{~nm})$ particles in base fluid. Nanofluids have very high thermal conductivity at very low nanoparticle concentrations and exhibit considerable enhancement of convection (Yang et al. [9]).

Several numerical methods have been proposed for the simulation of nanofluids. In this paper we present a boundary element method based algorithm for simulation of flow and heat transfer of nanofluids. We formulate the Navier-Stokes equations in velocity-vorticity form and couple them with the energy conservation equation. Special consideration is given to the determination of vorticity boundary conditions.

\section{Governing equations}

The governing equations are written using effective properties of the nanofluid. These are: density $\rho_{n f}$, dynamic viscosity $\mu_{n f}$, heat capacitance $\left(c_{p}\right)_{n f}$, thermal expansion coefficient $\beta_{n f}$ and thermal conductivity $k_{n f}$, where subscript $n f$ is used to denote effective (i.e. nanofluid) properties. The properties are all assumed constant throughout the flow domain. Pure fluid properties will be denoted by the subscript $f$. The properties are estimated using models (Oztop and Abu-Nada [3]) and depend on the nanoparticle volume fraction $\varphi$.

The nondimensional steady velocity-vorticity formulation of Navier-Stokes equations for simulation of nanofluids consists of the kinematics equation, the vorticity transport equation and the energy equation, relating the velocity $\vec{v}$, vorticity $\vec{\omega}$ and temperature $T$ fields. These equations may be written in the following way (Ravnik et al. [5]):

$$
\begin{gathered}
\nabla^{2} \vec{v}+\vec{\nabla} \times \vec{\omega}=0, \\
(\vec{v} \cdot \vec{\nabla}) \vec{\omega}=(\vec{\omega} \cdot \vec{\nabla}) \vec{v}+\operatorname{Pr} \frac{\mu_{n f}}{\mu_{f}} \frac{\rho_{f}}{\rho_{n f}} \nabla^{2} \vec{\omega}-\operatorname{Pr} R a \frac{\beta_{n f}}{\beta_{f}} \vec{\nabla} \times T \vec{g}, \\
(\vec{v} \cdot \vec{\nabla}) T=\frac{k_{n f}}{k_{f}} \frac{\left(\rho c_{p}\right)_{f}}{\left(\rho c_{p}\right)_{n f}} \nabla^{2} T .
\end{gathered}
$$

The flow and heat transfer of a nanofluid is thus defined by specifying the pure fluid Rayleigh and Prandtl number values. They are defined as

$$
R a=\frac{g_{0} \beta_{f} \Delta T L^{3} \rho_{f}\left(\rho c_{p}\right)_{f}}{\mu_{f} k_{f}}, \quad \operatorname{Pr}=\frac{\mu_{f} c_{p}}{k_{f}} .
$$

We consider water $\left(c_{p}=4179 \mathrm{~J} / \mathrm{kgK}, \rho=997.1 \mathrm{~kg} / \mathrm{m}^{3}, k=0.613 \mathrm{~W} / \mathrm{mK}\right.$, $\beta=21 \cdot 10^{-5} \mathrm{~K}^{-1}, \mu=0.912 \mathrm{~mm}^{2} / \mathrm{s}$ ) and $\mathrm{Al}_{2} \mathrm{O}_{3}$ nanofluid with particle volume fraction $\varphi=0.1$ and $\varphi=0.2$. The nanofluid properties are evaluated using models (Ravnik and Škerget [4]). These yield for the $\varphi=0.1 \mathrm{Al}_{2} \mathrm{O}_{3}$ nanofluid: $c_{p}=3132 \mathrm{~J} / \mathrm{kgK}, \rho=1294 \mathrm{~kg} / \mathrm{m}^{3}, k=0.807 \mathrm{~W} / \mathrm{mK}, \beta=14.82 \cdot 10^{-5} \mathrm{~K}^{-1}$, $\mu=1.187 \mathrm{~mm}^{2} / \mathrm{s}$ and for $\varphi=0.2 \mathrm{Al}_{2} \mathrm{O}_{3}$ nanofluid: $c_{p}=2476 \mathrm{~J} / \mathrm{kgK}$, $\rho=1592 \mathrm{~kg} / \mathrm{m}^{3}, k=1.047 \mathrm{~W} / \mathrm{mK}, \beta=10.95 \cdot 10^{-5} \mathrm{~K}^{-1}, \mu=1.593 \mathrm{~mm}^{2} / \mathrm{s}$. 


\section{Numerical procedure}

The governing equations were solved for heat and fluid flow by an in-house boundary element based algorithm (Ravnik et al. $[5,6,10])$. The algorithm solves the velocity-vorticity formulation of Navier-Stokes equations. It requires known velocity and temperature boundary conditions, while the boundary conditions for vorticity are unknown and are calculated as a part of the algorithm.

In the first step, the algorithm estimates boundary vorticity values using single domain BEM on the kinematics Equation (1). This step is described in detail in Section 3.1. Secondly, using sub-domain BEM solution of the kinematics Equation (1) the velocity in the domain is calculated. Thirdly, the energy Equation (3) is solved for domain temperature values using sub-domain BEM. Lastly, the vorticity transport Equation (2) is solved for domain vorticity values using sub-domain BEM. The procedure is repeated until convergence is achieved. Under-relaxation is used. A value of 0.1 is used for problems with low Rayleigh number value and 0.01 for problems with high Rayleigh number value.

The singular boundary integral representation for the velocity vector can be formulated by using the Green theorems for scalar functions, or weighting residuals technique. Following Wu and Thompson [8], Škerget et al. [7] derived the following integral form of the kinematics equation, employing the derivatives of the fundamental solution:

$$
c(\vec{\xi}) \vec{v}(\vec{\xi})+\int_{\Gamma} \vec{v}\left(\vec{n} \cdot \vec{\nabla} u^{\star}\right) d \Gamma=\int_{\Gamma} \vec{v} \times\left(\vec{n} \times \vec{\nabla} u^{\star}\right) d \Gamma+\int_{\Omega}\left(\vec{\omega} \times \vec{\nabla} u^{\star}\right) d \Omega,
$$

where $u^{\star}=u^{\star}(\vec{\xi}, \vec{r})$ is the elliptic Laplace fundamental solution, $\vec{\xi}$ is the source point on boundary $\Gamma, \vec{r}$ integration point in domain $\Omega$ (including $\Gamma$ ), $c(\vec{\xi})$ geometry coefficient and $\vec{n}$ outward pointing normal to the boundary. Geometry coefficient can be generally computed as $\Theta / 4 \pi$, where $\Theta$ is the internal solid angle at point $\vec{\xi}$ in steradians. The Laplace fundamental solution is $u^{\star}(\vec{\xi}, \vec{r})=1 /(4 \pi|\vec{\xi}-\vec{r}|)$.

The integral forms of vorticity transport and energy equation are

$$
\begin{aligned}
& c(\vec{\xi}) \omega_{j}(\vec{\xi})+\int_{\Gamma} \omega_{j} \vec{\nabla} u^{*} \cdot \vec{n} d \Gamma=\int_{\Gamma} u^{*} q_{j} d \Gamma \\
& +\frac{1}{\operatorname{Pr}} \frac{\mu_{f}}{\mu_{n f}} \frac{\rho_{n f}}{\rho_{f}}\left(\int_{\Gamma} \vec{n} \cdot\left\{u^{*}\left(\vec{v} \omega_{j}-\vec{\omega} v_{j}\right)\right\} d \Gamma-\int_{\Omega}\left(\vec{v} \omega_{j}-\vec{\omega} v_{j}\right) \cdot \vec{\nabla} u^{*} d \Omega\right) \\
& \quad-R a \frac{\beta_{n f}}{\beta_{f}} \frac{\mu_{f}}{\mu_{n f}} \frac{\rho_{n f}}{\rho_{f}} \int_{\Gamma}\left(u^{\star} T \vec{g} \times \vec{n}\right)_{j} d \Gamma \\
& \quad-R a \frac{\beta_{n f}}{\beta_{f}} \frac{\mu_{f}}{\mu_{n f}} \frac{\rho_{n f}}{\rho_{f}} \int_{\Omega}\left(T \vec{\nabla} \times u^{\star} \vec{g}\right)_{j} d \Omega \\
& c(\vec{\xi}) T(\vec{\xi})+\int_{\Gamma} T \vec{\nabla} u^{*} \cdot \vec{n} d \Gamma=\int_{\Gamma} u^{*} T_{q} d \Gamma \\
& +\frac{k_{f}}{k_{n f}} \frac{\left(\rho c_{p}\right)_{n f}}{\left(\rho c_{p}\right)_{f}}\left(\int_{\Gamma} \vec{n} \cdot\left\{u^{*}(\vec{v} T)\right\} d \Gamma-\int_{\Omega}(\vec{v} T) \cdot \vec{\nabla} u^{*} d \Omega\right)
\end{aligned}
$$


In the subdomain BEM method we make a mesh of the entire domain $\Omega$ and name each mesh element a subdomain. Equations (5), (6) and (7) are written for each of the subdomains. In order to obtain a discrete version of the equations, we use shape functions to interpolate field functions and flux across the boundary and inside of the subdomain.

\subsection{Vorticity boundary conditions for an arbitrary 3D surface}

Several different approaches have been proposed for the determination of vorticity on the boundary. We propose the usage of singular integral kinematics equation. In this work, we extend the approach for determining boundary vorticity on an arbitrary 3D surface.

The normal component of vorticity at the boundary is usually known. If we consider a wall, then the velocity at the wall is either zero or we know the value of slip velocity. Thus, the normal component of vorticity may be calculated directly from the known velocity distribution at the wall. This is possible due to the fact that in order to calculate the normal component of vorticity only tangential components of the velocity are needed. The same reasoning applies at the inlets and outlets as well, since the velocity profile is known there. In the case of symmetry or free slip boundary conditions, the flux of normal component of vorticity is zero. This can be used in the vorticity transport equation and as a result, the normal component of boundary vorticity can be calculated there.

For an arbitrary surface, such as the cylinder in our case, the normal component of vorticity is calculated using Cartesian vorticity components and the unit normal to the surface, i.e. $\omega_{n}=\vec{n} \cdot \vec{\omega}=\sum_{i} n_{i} \omega_{i}$, where $i=x, y, z$. Since we know that $\omega_{n}=0$ at the no-slip surface and $\vec{n}$ changes along the surface, we propose the following strategy to find $\omega_{x}, \omega_{y}$ and $\omega_{z}$.

To obtain discrete form of integral Equation (5) we divide computational domain $\Omega$ into domain elements and its boundary $\Gamma$ into boundary elements. Domain elements used are hexahedra with 27 nodes enabling quadratic interpolation. Boundary elements used are sides of domain hexahedra with 9 nodes and also enable quadratic interpolation. A function, e.g. temperature, is interpolated over a boundary elements as $T=\sum \Xi_{i} T_{i}$, inside each domain element as $T=\sum \Phi_{i} T_{i}$. Functions $\Xi_{i}$ and $\Phi_{i}$ are interpolation functions.

After a choice of the source point $\vec{\xi}$ in (5) has been made and interpolation of functions used, the integrals in (5) depend only on the geometry and the fundamental solution. They may be calculated and stored in matrices. The boundary integral on the left hand side are stored in the $[H]$ matrix, the boundary integral on the right hand side in the $\left[\vec{H}^{t}\right]$ matrix and the domain integrals on the right hand side are the $[\vec{D}]$ matrices. For each source point a row in the matrices is calculated:

$$
[H]=\int_{\Gamma} \Xi_{i}\left(\vec{n} \cdot \vec{\nabla} u^{\star}\right) d \Gamma, \quad\left[\vec{H}^{t}\right]=\int_{\Gamma} \Xi_{i}\left(\vec{n} \times \vec{\nabla} u^{\star}\right) d \Gamma
$$




$$
[\vec{D}]=\int_{\Omega} \Phi_{i} \vec{\nabla} u^{\star} d \Omega .
$$

The $[H]$ matrix holds integrals of normal derivatives of the fundamental solution, $\left[\vec{H}^{t}\right]$ tangential derivatives and $[\vec{D}]$ the gradient of the fundamental solution. Thus the discrete version of Equation (5) may be written as

$$
[H]\{\vec{v}\}=\{\vec{v}\} \times\left[\vec{H}^{t}\right]+\{\vec{\omega}\} \times[\vec{D}],
$$

where curly brackets denote vectors of nodal values of field functions. In order to obtain a system of linear equations, the source point is placed into all boundary nodes. Thus the number of rows in all matrices is equal to the number of boundary nodes. The number of columns in $[H]$ and $\left[\vec{H}^{t}\right]$ is also equal to the number of boundary nodes since they are multiplied by boundary velocity values. On the other hand, the number of columns in $[\vec{D}]$ is equal to the number of all nodes, as $[\vec{D}]$ is multiplied by vorticity in the domain and on the boundary.

In order to use Equation (10) to solve for boundary vorticity values we decompose the vorticity vector into two parts in the following way $\left\{\omega_{i}\right\}=$ $\left\{\omega_{i}\right\}_{\Gamma}+\left\{\omega_{i}\right\}_{\Omega^{\prime}}$. In the vector $\left\{\omega_{i}\right\}_{\Gamma}$ only the boundary vorticity values are non-zero and in the vector $\left\{\omega_{i}\right\}_{\Omega^{\prime}}$ only the domain vorticity values are nonzero. The subscript $\Gamma$ stands for boundary nodes only and $\Omega^{\prime}$ stands for interior nodes only (without boundary nodes). Furthermore, one must set up the system in such a way, that the system matrix is non-singular. Since we are dealing with boundary element method, the system matrix may contain a normal derivative of the fundamental solution for the integral kernel. The integral kernel in the matrices $\left[D_{x}\right],\left[D_{y}\right],\left[D_{z}\right]$ are the components of the gradient of the fundamental solution. The normal derivative may be written as $\left[n_{x}\right]\left[D_{x}\right]+\left[n_{y}\right]\left[D_{y}\right]+\left[n_{z}\right]\left[D_{z}\right]=[\vec{n}] \cdot[\vec{D}]$, where $\left[n_{x}\right],\left[n_{y}\right]$ and $\left[n_{z}\right]$ are diagonal matrices of unit normal vector components $\vec{n}=\left(n_{x}, n_{y}, n_{z}\right)$ for each boundary source point. $[\vec{n}]$

To obtain such a system, we perform a vector product of (10) by normal vector

$$
[H]([\vec{n}] \times\{\vec{v}\})=[\vec{n}] \times\left(\{\vec{v}\} \times\left[\vec{H}^{t}\right]+\{\vec{\omega}\} \times[\vec{D}]\right),
$$

and after using $[\vec{n}] \times(\{\vec{\omega}\} \times[\vec{D}])=([\vec{n}] \cdot[\vec{D}])\{\vec{\omega}\}-[\vec{D}]\left\{\omega_{n}\right\}$ and $\{\vec{\omega}\}=$ $\{\vec{\omega}\}_{\Gamma}+\{\vec{\omega}\}_{\Omega^{\prime}}$ and rearranging we obtain

$$
\begin{aligned}
& ([\vec{n}] \cdot[\vec{D}])\{\vec{\omega}\}_{\Gamma}=[\vec{D}]\left\{\omega_{n}\right\}-([\vec{n}] \cdot[\vec{D}])\{\vec{\omega}\}_{\Omega^{\prime}} \\
& -\left([\vec{n}] \cdot\left[\vec{H}^{t}\right]\right)\{\vec{v}\}+\left[\vec{H}^{t}\right]\left\{v_{n}\right\}+[H]([\vec{n}] \times\{\vec{v}\}) .
\end{aligned}
$$

In (12) all three equations for individual components of boundary vorticity are non-singular. However, they can only be used to solve for tangential components of the boundary vorticity, since the equation for normal component of boundary vorticity is identically equal to zero. This can be seen, if we consider a boundary located in plane $y-z$ with the unit normal $\vec{n}=\{1,0,0\}$. In this case $\omega_{x}$ is the normal component of the vorticity and $v_{x}$ is the normal component of velocity. 
We observe that all terms in the Equation (12) for $\omega_{x}$ are either zero or cancel each other. Thus, the equation is identically equal to zero and it can not be used for the solution of the normal component of vorticity.

Finally, the algorithm for determining the boundary vorticity is as follows. At each source point, which is located at the boundary, compare $\left|n_{x}\right|,\left|n_{y}\right|$ and $\left|n_{z}\right|$ to find the largest component of the normal vector. Use Equations (12) to find the other two components of vorticity and use equation $\omega_{n}=\vec{n} \cdot \vec{\omega}$ and the known $\omega_{n}$ to find the last boundary vorticity component. For example, if $\left|n_{x}\right|>\left|n_{y}\right|$ and $\left|n_{x}\right|>\left|n_{z}\right|$ then solve (12) for $\omega_{y}$ and $\omega_{z}$ and solve $\omega_{n}=\vec{n} \cdot \vec{\omega}$ for $\omega_{x}$.

\section{Problem description}

A heated cylinder is inserted into an enclosure with four cooled walls. Front and back walls are perfectly insulated (adiabatic). All walls have a no-slip boundary condition applied for velocity. The heat is transferred from the cylinder to the fluid causing density changes that result in buoyancy forces. Natural convection develops - the fluid rises around the cylinder and transports heat towards the cold walls. The heat flux depends on the type of fluid, the shape of the cylinder and the orientation of the enclosure with respect to gravity.

The centre of the cylinder is located at the centre of the enclosure. The shape of the base of the cylinder is an ellipse with major semi-axis $a$ and minor semi axis $b$. The are defined as

$$
a=0.2 L, \quad b=a \sqrt{1-e^{2}},
$$

where $e$ is the eccentricity of the ellipse and the length of the cylinder is $L$. The enclosure is cubic with a volume of $L^{3}$. It is tilted with respect to gravity with an angle of $\gamma$. The temperature of the cylinder is constant $T_{h}$ and the temperature of the cold walls is also constant, $T_{c}$.

In order to validate the numerical model and assess mesh computational quality, we performed simulations using air as the working fluid and compared results to Kim et al. [2], who studied natural convection of air in a square enclosure with a circular cylinder inserted in 2D.

Comparison is done for Rayleigh number values $R a=10^{3}-10^{6}$. The flow regime is laminar and steady. Heat transfer from the cylinder into the fluid is measure in terms of the Nusselt number. Three computational meshes are considered. Comparison is presented in Table 1. We observe good agreement with the results of Kim et al. [2], who studied the 2D case. Looking at the results on the fine mesh, we observe all Nusselt number values are within $1 \%$ of the Kim's results. Based on this, we decided to use the fine mesh for all simulations presented in the results section.

\section{Results}

We considered several Rayleigh number values as well as different angles of inclination versus gravity. The elliptical shape causes a change in flow regime 


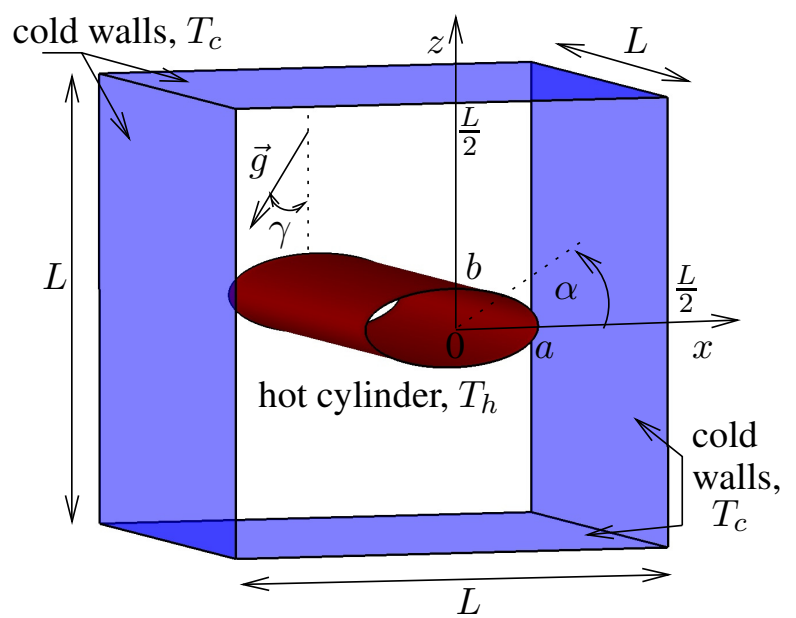

Figure 1: Computational domain and coordinate system with boundary conditions. The angle $\alpha$ measures a location around the circumference of the cylinder. The angle $\gamma$ measures the tilt of the enclosure with respect to the gravity vector. In a 3D simulation the front and back walls ( $y=0$ and $y=L$ ) are adiabatic and have no-slip boundary condition for velocity. In a $2 \mathrm{D}$ simulation, the font and back walls have symmetry boundary conditions applied.

Table 1: Validation of the numerical method. Average Nusselt number values at the hot cylinder for different values of the Rayleigh number are compared with the results of Kim et al. [2]. Simulations are performed in 2D for a circular cylinder $(e=0)$ on several meshes using air $(\operatorname{Pr}=0.7)$ as the working fluid. Coarse mesh had 14.4, fine 32.0 and very fine 39.4 thousands nodes.

\begin{tabular}{|c|c|c|c|c|}
\hline Mesh & $10^{3}$ & $10^{4}$ & $10^{5}$ & $10^{6}$ \\
\hline Very fine & 5.041 & 5.133 & 7.756 & 14.020 \\
Fine & 5.041 & 5.133 & 7.779 & 14.080 \\
Coarse & 5.042 & 5.135 & 7.834 & 14.275 \\
\hline Kim et al. [2] & 5.093 & 5.108 & 7.767 & 14.110 \\
\hline
\end{tabular}


when tilted against gravity. To illustrate this point we present temperature contours and streamlines in Figures 2 and 3. At $R a=10^{4}$ the heat transfer is conduction dominated and thus temperature contours keep the elliptical shape of the cylinder. Streamlines reveal a symmetrical flow field, with a vortex on both sides of the cylinder. Vortex centres are located approximately on a diagonal line going through the enclosure from the top-left corner to the bottom-right corner.
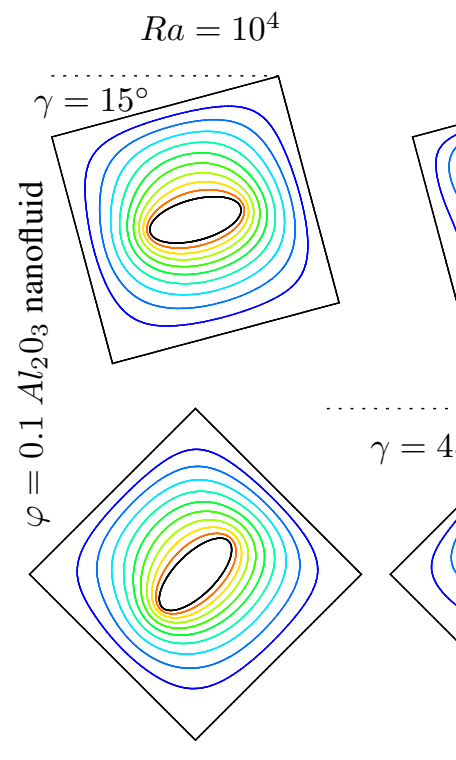

$$
R a=10^{5}
$$$$
R a=10^{6}
$$
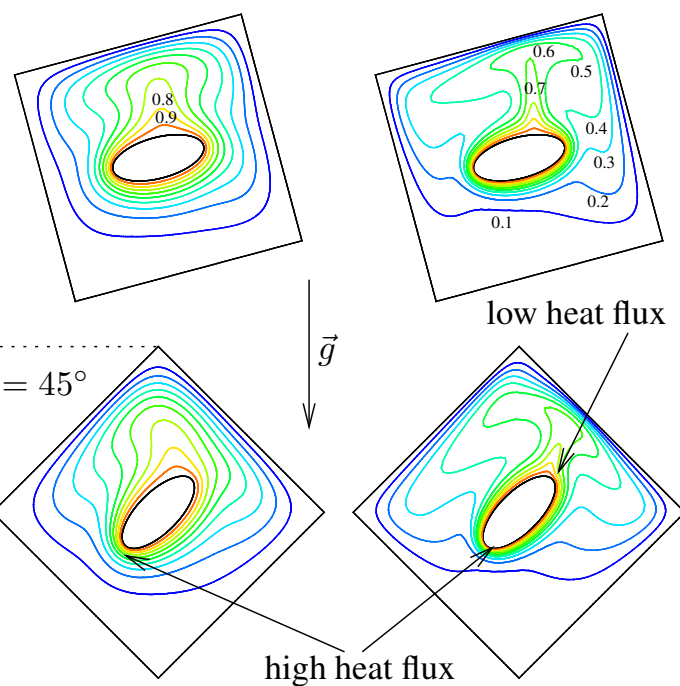

Figure 2: Temperature contours of $\varphi=0.1 \mathrm{Al}_{2} \mathrm{O}_{3}$ nanofluid in 2D simulation with elliptical cylinder for $R a=10^{4} \ldots 10^{6}$. Top panels show results at $\gamma=15^{\circ}$, while bottom panels present $\gamma=45^{\circ}$. Nine contour levels are shown with values between 0.1 and 0.9 in steps of 0.1 .

When we look at the convection dominated cases $\left(R a=10^{5}\right.$ and $\left.R a=10^{6}\right)$ we observe that the symmetry is lost. Raising the tilt of the enclosure causes movement of the line, which divides both vortices. The location of this dividing line is important, as flow stagnates there and causes that area of the cylinder to have the lowest heat transfer. We observe that the line is located at the point of the cylinder, which is highest (has the larger $z$ coordinate). Thus tilting the enclosure against gravity (raising $\gamma$ ) moves the low heat transfer zone away from the top of the cylinder $\left(\alpha=90^{\circ}\right)$ towards the side $\left(\alpha=0^{\circ}\right)$.

This can be also observed when we look at the heat flux distribution around the circumference of the cylinder in Figure 4. On the other hand, in the conduction regime $R a \leq 10^{4}$, the tilt against gravity does not affect the heat flux.

The heat flux distribution around the cylinder features two peaks at the sides of the enclosure. Tilting the enclosure increases the heat transfer around most of the 

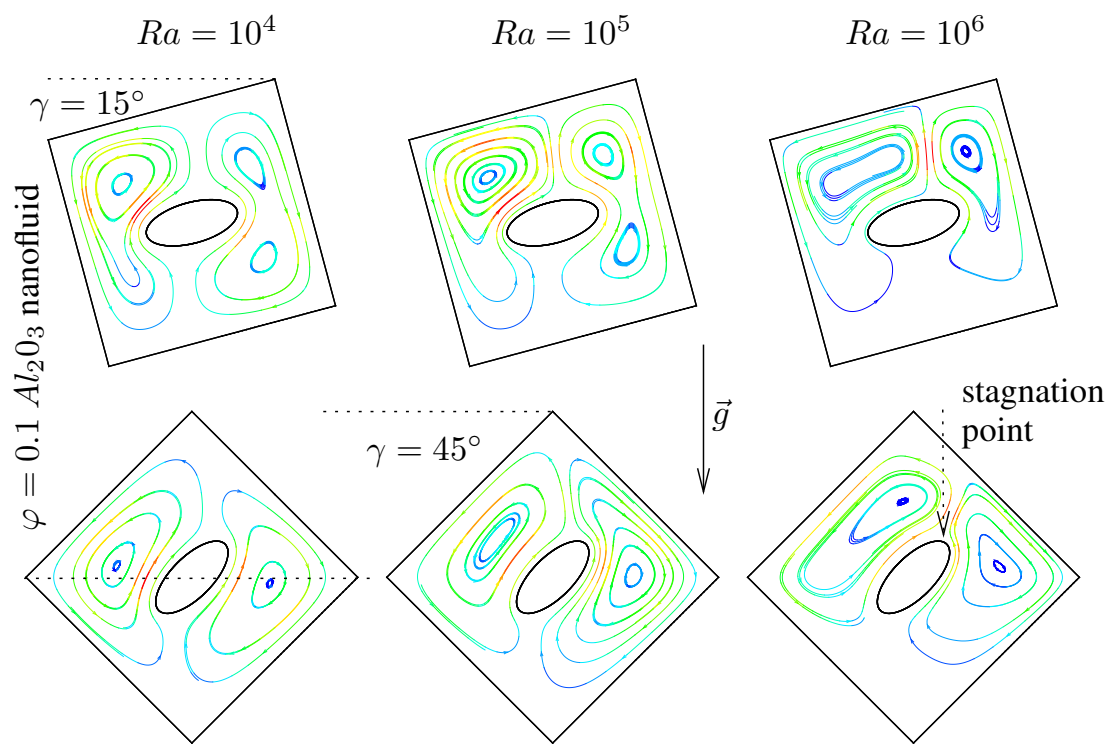

Figure 3: Streamlines $\varphi=0.1 \mathrm{Al}_{2} \mathrm{O}_{3}$ nanofluid in 2D simulation with elliptical cylinder for $R a=10^{4} \ldots 10^{6}$. Top panels show results at $\gamma=15^{\circ}$, while bottom panels present $\gamma=45^{\circ}$. Colour denotes velocity magnitude.

Table 2: Average Nusselt number values for an elliptical cylinder obtain in 2D and 3D simulations.

\begin{tabular}{|c|c|ccc|ccc|}
\hline \multicolumn{2}{|c|}{$R a$} & $10^{3}$ & $10^{4}$ & $10^{5}$ & $10^{3}$ & $10^{4}$ & $10^{5}$ \\
\hline & $\gamma$ & $\varphi=0.1, A_{2} O_{3}$ & nanofluid & $\varphi=0.2, A l_{2} O_{3}$ nanofluid \\
\hline 2D & 0 & 6.686 & 6.758 & 9.886 & 8.674 & 8.697 & 10.742 \\
2D & 15 & 6.686 & 6.763 & 9.935 & 8.674 & 8.699 & 10.750 \\
2D & 30 & 6.686 & 6.777 & 10.068 & 8.674 & 8.705 & 10.797 \\
2D & 45 & 6.686 & 6.799 & 18.434 & 8.674 & 8.713 & 10.991 \\
\hline 3D & 0 & 6.684 & 6.742 & 9.598 & 8.672 & 8.691 & 10.439 \\
3D & 15 & 6.684 & 6.746 & 9.644 & 8.672 & 8.693 & 10.456 \\
3D & 30 & 6.684 & 6.759 & 9.755 & 8.672 & 8.697 & 10.511 \\
3D & 45 & 6.685 & 6.776 & 9.962 & 8.672 & 8.704 & 10.683 \\
\hline
\end{tabular}



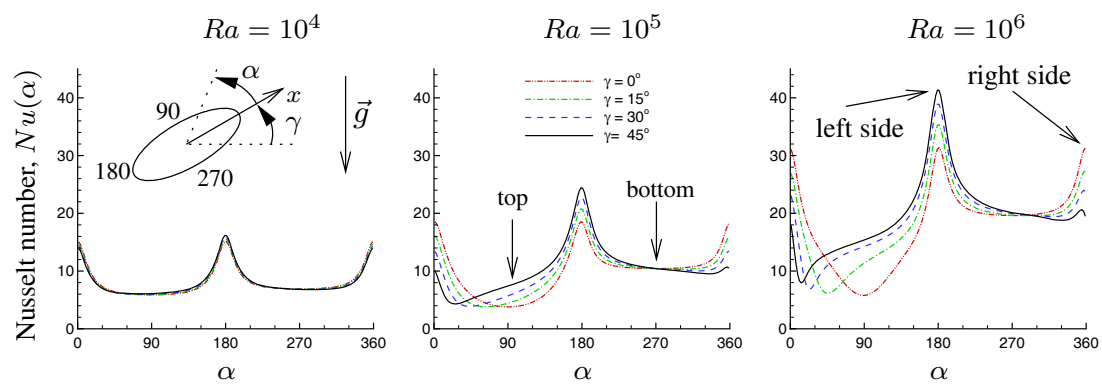

Figure 4: Heat flux around the circumference of the ellipsoidal cylinder expressed as Nusselt number. Results of 2D simulations of $\varphi=0.2 \mathrm{Al}_{2} \mathrm{O}_{3}$ nanofluid are shown for $R a=10^{4}$ (left), $R a=10^{5}$ (left) and $R a=10^{6}$ (right). Results for different angles $\gamma$ against gravity are presented.

cylinder apart from the area around $\alpha=0^{\circ}$, where the heat flux is decreased. The highest heat transfer is found at bottom left side of the cylinder $\left(\alpha=180^{\circ}\right)$.

The heat transfer averages expressed as Nusselt number values are given in Table 2 for 2D and 3D simulations. The data reveals heat transfer enhancement when using nanofluids instead of pure water (for zero tilt the $N u$ for pure water as $N u=5.078$ at $R a=10^{3}, N u=5.293$ at $R a=10^{4}$ and $N u=8.936$ at $\left.R a=10^{5}\right)$.

The enhancement is largest when conduction is the dominating heat transfer mechanism $\left(R a \leq 10^{4}\right)$, where we observe about $\approx 30 \%$ increase in heat flux for $\varphi=0.1$ nanofluid and about $\approx 70 \%$ increase when using $\varphi=0.2$ nanofluid. As convection becomes important, relative enhancement is smaller, since the fluid properties play a less important role in determining heat flux.

Comparison of 2D and 3D simulations shows, that 3D simulations yield slightly lower heat transfer rates. Thus we may conclude, that this type of problem may be investigated in $2 \mathrm{D}$. The $3 \mathrm{D}$ effect will be more important in the case of unsteady and turbulent natural convection.

\section{Summary}

The paper presents a boundary element based numerical method for simulation of flow and heat transfer of nanofluids. The Navier-Stokes equations are used in velocity-vorticity form. Special consideration was given to the algorithm for determining the boundary vorticity values at an arbitrary 3D boundary surface, which is based on the boundary-domain integral kinematics equation.

The developed method has been used to study nanofluid heat transfer enhancement for the case of a cylinder in an enclosure. An elliptical cylinder was considered for various Rayleigh number values and inclinations against gravity. We found that the use of nanofluid enhances heat transfer the most in the case, where the majority of the heat is transferred by conduction. In cases, where 
convection is the dominant heat transfer mechanism, the heat transfer enhancement due to the use of a nanofluid is lower. Tilting the elliptical cylinder against gravity increases the heat transfer rate and changes the flow structure. The increase is small in flows, where conduction dominates, while it is larger in convection dominated flows. Furthermore it changes the locations on the cylinder, where lowest heat transfer is observed. Comparison of 2D and 3D simulations shows, that 3D simulations yield slightly lower heat transfer rates. The difference is very small for conduction dominated flows, while in convection dominated flows it is larger. As the differences are small, 2D simulations may be used to analysis such problems.

\section{References}

[1] S. U. S. Choi. Enhancing thermal conductivity of fluids with nanoparticles. Develop. Appl. Non Newtonian Flows, 66:99-106, 1995.

[2] B. S. Kim, D. S. Lee, M. Y. Ha and H. S. Yoon. A numerical study of natural convection in a square enclosure with a circular cylinder at different vertical locations. Int. J. Heat Mass Transfer, 51:1888-1906, 2008.

[3] H. F. Oztop and E. Abu-Nada. Natural convection of water-based nanofluids in an inclined enclosure with a heat source. Int. J. Heat Fluid Flow, 29:13261336, 2008.

[4] J. Ravnik and L. Škerget. A numerical study of nanofluid natural convection in a cubic enclosure with a circular and an ellipsoidal cylinder. Int. J. Heat Mass Transfer, 89:596-605, 2015.

[5] J. Ravnik, L. Škerget and M. Hriberšek. Analysis of three-dimensional natural convection of nanofluids by BEM. Eng. Anal. Bound. Elem., 34:1018-1030, 2010.

[6] J. Ravnik, L. Škerget and Z. Žunič. Velocity-vorticity formulation for 3D natural convection in an inclined enclosure by BEM. Int. J. Heat Mass Transfer, 51:4517-4527, 2008.

[7] L. Škerget, M. Hriberšek and Z. Žunič. Natural convection flows in complex cavities by BEM. Int. J. Num. Meth. Heat \& Fluid Fl., 13:720-735, 2003.

[8] J. C. Wu and J. F. Thompson. Numerical solutions of time-dependent incompressible Navier-Stokes equations using an integro-differential formulation. Comput. Fluids, 1:197-215, 1973.

[9] Y. Yang, Z. G. Zhang, E. A. Grulke, W. B. Anderson and G. Wu. Heat transfer properties of nanoparticle-in-fluid dispersions (nanofluids) in laminar flow. Int. J. Heat Mass Transfer, 48:1107-1116, 2005.

[10] Z. Žunič, M. Hriberšek, L. Škerget and J. Ravnik. 3-D boundary elementfinite element method for velocity-vorticity formulation of the Navier-Stokes equations. Eng. Anal. Bound. Elem., 31:259-266, 2007. 